\title{
Intravenous Immunoglobulin for the Treatment of COVID-19: A Promising Tool
}

\author{
Vasilios Tzilas ${ }^{a, b}$ Effrosyni Manalic Spyridon Papiris ${ }^{c}$ Demosthenes Bouros ${ }^{a, b}$ \\ ${ }^{a}$ First Academic Department of Pneumonology, Interstitial Lung Diseases Unit, Hospital for Diseases of the Chest, \\ "Sotiria," Medical School, National and Kapodistrian University of Athens, Athens, Greece; ${ }^{\mathrm{b}}$ Athens Medical Center, \\ Athens, Greece; 'Second Pulmonary Medicine Department, Attikon University Hospital, Medical School, National \\ and Kapodistrian University of Athens, Athens, Greece
}

The current pandemic caused by the novel coronavirus, severe acute respiratory syndrome coronavirus 2 (SARS-CoV-2), continues to relentlessly rage across the globe, representing a major health issue. The heavy burden that SARS-CoV-2 is posing to the health systems across the globe hampers the care of all patients $[1,2]$. The devastating effects of coronavirus disease 2019 (COVID-19) are due to its high infection rate and mortality. Pneumonia is the major cause of morbidity and mortality in COVID-19. Unfortunately, the lack of an effective antiviral agent and vaccine further complicates the situation and calls for further intensification in research. In parallel with the development of new agents, it is prudent to study the efficacy of existing therapeutic options with an acceptable safety profile. The administration of intravenous immunoglobulin (IVIG) represents such an example. IVIG is a blood product prepared from the serum pooled from thousands of healthy donors. The major component of IVIG preparations is the serum IgG fraction consisting mainly of IgG1 and IgG2 subclasses [3]. Traces of IgA and IgM can also be found. Initially, the rationale for its use was straightforward as it was administered to patients with immunodeficiency due to hypoglobulinemia. Since then it has been shown that IVIG ex-

karger@karger.com

(C) 2020 S. Karger AG, Basel

www.karger.com/res

Karger erts pleiotropic immunomodulating actions, involving both innate and adaptive immunity, and it has been used in a variety of diseases such as hematologic, neuromuscular, rheumatologic, ophthalmologic, and dermatologic disorders [4]. In the context of COVID-19, the actual role of IVIG is not to boost the immune system, but through its immunomodulatory effect to suppress a hyperactive immune response that is seen in some patients. This overwhelming response, which is vaguely described as cytokine storm syndrome, ends up being the major cause of lung injury [5]. This highlights the importance of selecting the right patient and intervening at the right moment.

In this issue of Respiration, Herth et al. [6] provide valuable information regarding the use of IVIG for the treatment of COVID-19 in 12 patients (9 male) with an average age of 50 years (range 23-74 years) and severe COVID-19. The severity is reflected on median and mean APACHE II scores of 13 and 16, respectively. This was a retrospective study including patients from three hospitals, two from the United States of America and one from Germany. Patients who received IVIG were at different stages of their illness, specifically within 3 days of symptom onset, after initiation of mechanical ventilation, and after prolonged ventilation and stay in the intensive care 
unit. However, it must be stressed that the majority of patients $(8 / 12,75 \%)$ were mechanically ventilated and almost half of them $(5 / 12,41.7 \%)$ received extracorporeal membrane oxygenation support. The total IVIG dose ranged from 0.5 to $2.0 \mathrm{~g} / \mathrm{kg}$ (median $1.25 \mathrm{~g} / \mathrm{kg}$ ) and was administered over 1-4 daily doses, with the most common regimen received being $0.5 \mathrm{~g} / \mathrm{kg}$ daily for 3 days. The administration of IVIG was well tolerated.

The promising result from this study is that all patients survived hospital discharge. Notably, in some cases the authors report that the initiation of IVIG resulted in immediate and striking improvement in terms of clinical symptoms, laboratory examinations, and imaging. The time of administration seems to play an important role in achieving a successful outcome. Early administration of IVIG (within 3 days of admission) was correlated with a significantly shorter length of hospital stay (median 7 days, range 3-14 days). On the other hand, "late" administration of IVIG ( $>7$ days since admission) was correlated with a significantly longer hospital stay (median 33 days, range $8-48$ days, $p=0.03$ ). The above suggests that the greatest benefit of IVIG in the setting of COVID-19 is achieved with early administration. However, this should not discourage the use of IVIG in patients with a more prolonged course. In the series by Herth et al. [6], administration of IVIG in 2 patients with protracted illness resulted in significant improvement and eventually hospital discharge. The main limitations of the current study, as the authors report, are its retrospective nature, the relatively low number of patients, and the lack of a comparator arm. Nevertheless, the results are encouraging and call for further studies. Currently, according to ClinicalTrials. gov there are 37 studies of IVIG in patients with COVID-19, of which 23 are in the recruitment phase.

The use of IVIG has not been found to be beneficial in hospitalized patients with influenza A or B infection [7]. This should not discourage further research in the field of viral pneumonias and COVID-19 in particular. In a retrospective study of 58 cases with severe or critical illness due to COVID-19, early administration of IVIG was associated with reduced ventilator use, reduced hospital and intensive care unit length of stay, and improved 28day mortality [8]. Given the immunomodulatory effect of IVIG, it is important to properly select patients for this kind of treatment. In respiratory viral infections, an effective immune response is mandatory to control infection. In some case the response of the immune system is overwhelming and becomes the main cause of lung injury. These are the patients with severe COVID-19, characterized with lymphopenia and the so-called inflammatory cytokine storm $[5,9,10]$. Furthermore, besides selecting the appropriate patients, timely administration of IVIG (and any immunomodulating agent in general) is crucial. The initiation of the cytokine storm takes place 5-7 days after initiation of symptoms and represents the time window in which immunomodulation is likely to be most beneficial [5]. The importance of timely administration of IVIG has been shown in the current study by Herth et al. [6] as well. Continuing research is essential in order to better understand the pathogenesis of COVID-19 and therefore optimize management. In the meantime and given the fact that discovery of a "silver bullet" against SARS-CoV-2 is unlikely in the near future, it is equally important to apply all the hard-earned experience and knowledge that have been acquired over several decades in order to provide the optimal outcome for these patients.

\section{Statement of Ethics}

As an invited editorial the paper is exempt from ethics committee approval.

\section{Conflict of Interest Statement}

None of the authors have any conflicts of interest to disclose.

\section{Funding Sources}

None.

\section{Author Contributions}

V. Tzilas and D. Bouros wrote the manuscript. All authors read and accepted the manuscript.

\section{References}

1 Antoniou KM, Raghu G, Tzilas V, Bouros D. Management of patients with interstitial lung disease in the midst of the COVID-19 pandemic. Respiration. 2020;99(8):625-7.

2 Antoniou K, Bolaki M, Bibaki E, Vasarmidi E, Trachalaki A, Tsitoura E, et al. COVID19 alert. Do we know our enemy? Pneumon. 2020;33(1):25-7.

3 Schwab I, Nimmerjahn F. Intravenous immunoglobulin therapy: how does IgG modulate the immune system? Nat Rev Immunol. 2013 Mar;13(3):176-89.
Tzilas/Manali/Papiris/Bouros 
4 Quinti I, Mitrevski M. Modulatory Effects of Antibody Replacement Therapy to Innate and Adaptive Immune Cells. Front Immunol. 2017 Jun;8:697-697.

5 Buszko M, Park JH, Verthelyi D, Sen R, Young HA, Rosenberg AS. The dynamic changes in cytokine responses in COVID-19: a snapshot of the current state of knowledge. Nat Immunol. 2020 Oct;21(10):1146-51.
6 Herth FJF, Sakoulas G, Haddad F. Use of intravenous immunoglobulin for the treatment of COVID-19: retrospective case series. Respiration. doi: 10.1159/000511376.

7 Davey RT Jr, Fernández-Cruz E, Markowitz N, Pett S, Babiker AG, Wentworth D, et al.; INSIGHT FLU-IVIG Study Group. Anti-influenza hyperimmune intravenous immunoglobulin for adults with influenza A or B infection (FLU-IVIG): a doubleblind, randomised, placebo-controlled trial. Lancet Respir Med. 2019 Nov;7(11): 951-63.
8 Xie Y, Cao S, Dong H, Li Q, Chen E, Zhang $\mathrm{W}$, et al. Effect of regular intravenous immunoglobulin therapy on prognosis of severe pneumonia in patients with COVID-19. J Infect. 2020 Aug;81(2):318-56.

9 Vaninov N. In the eye of the COVID-19 cytokine storm. Nat Rev Immunol. 2020 May; 20(5):277-277.

10 Del Valle DM, Kim-Schulze S, Huang HH, Beckmann ND, Nirenberg S, Wang B, et al. An inflammatory cytokine signature predicts COVID-19 severity and survival. Nat Med. 2020 Oct;26(10):1636-43. 